\title{
Consumo de tabaco en poblaciones estigmatizadas: una revisión de la literatura en Pubmed

\author{
Tabaco en población estigmatizada
}

\author{
Tobacco consumption in stigmatized populations: \\ A review of the literature on PubMed \\ Tobacco in stigmatized populations
}

\author{
AdRIANA Bravo(1); MARÍA CARolina CABRERA ${ }^{(1)}$; LUISA FERnANDA GómeZ ${ }^{(1)}$; \\ ANDRÉS FELIPE PINTO(1); DIEGO ROSSELLI ${ }^{(2)}$
}

\section{Resumen}

INTRODUCCIÓN: las poblaciones de esquizofrénicos, homosexuales, presidiarios y trabajadores sexuales, están expuestas a factores de riesgo que afectan su morbimortalidad.

OBJETIVO: revisar la literatura indexada en PubMed para tratar de cuantificar el incremento del consumo de tabaco en estos grupos.

MATERIALES Y MÉTODOS: se diseñó una estrategia de búsqueda sensible para cada uno de los grupos de interés. Dos integrantes del equipo revisaron títulos y resúmenes independientemente para excluir los irrelevantes. Luego de conseguir los textos completos se tabuló la información de cada estudio.

RESULTADOS: de 1.894 referencias, se excluyeron 1.365 en un primer filtro, 91 no se consiguieron y 206 estudios, provenientes de 31 países, aportaron información (101 de esquizofrenia, 68 de población homosexual, 31 de presidiarios y 6 de trabajadores sexuales). Las razones de disparidad (IC $95 \%$ ) contra grupo control fueron: esquizofrénicos 3,34 (2,95 - 3,78); población homosexual 2,03 (1,84-2,26); presidiarios hombres 6,60 $(5,15-8,46)$, mujeres 9,72 $(5,95-15,90)$ y trabajadoras sexuales $26,3(6,7-103,2)$.

DISCUSIÓN: el consumo de tabaco es consistentemente elevado en estos grupos estigmatizados. Unido a otros estilos de vida poco saludables explican por qué sus riesgos en salud son mayores. Es necesario diseñar estrategias de intervención específicas para cada grupo.

Palabras clave: esquizofrenia, fumar, homosexualidad, prisioneros, prostitución, tabaco.

\section{Abstract}

INTRODUCTION: populations of schizophrenics, homosexuals, jail inmates, and sex workers are exposed to risk factors that affect their morbidity and mortality.

OBJECTIVE: to review the indexed literature available on PubMed, in order to try to quantify the increase in tobacco consumption among these groups.

MATERIALS AND METHODS: a sensitive search strategy was designed for each group of interest. Titles and abstracts were reviewed independently by two team members, in order to exclude irrelevant ones. After obtaining the full texts, the information from each study was tabulated.

RESULTS: of 1894 references, 1365 were excluded at first screening, 91 could not be obtained, and 206 studies, from 31 countries, supplied information (101 about schizophrenia, 68 about homosexual population, 31 about jail inmates, and 6 about sex workers). The odds ratios
(1)Estudiante de Medicina. Pontificia Universidad Javeriana. Bogotá, Colombia. (2) Profesor Asociado. Departamento de Epidemiología Clínica y Bioestadística. Facultad de Medicina Pontificia Universidad Javeriana. Bogotá, Colombia.

Correspondencia: Diego Rosselli, correo electrónico: diego.rosselli@gmail.com Recibido: 07/07/15. Aceptado: 17/07/15. 
(CI 95\%) with regard to the control group were: schizophrenics 3,34 (2,95 - 3,78); homosexual population 2,03 (1,84 - 2,26); male prison inmates $6,60(5,15-8,46)$, female inmates $9,72(5,95-15,90)$, and sex workers $26,3(6,7-103,2)$.

DISCUSSION: Tobacco consumption is consistently high in these stigmatized groups. Together with other unhealthy lifestyles, it explains why their health risks are greater. It is necessary to design group-specific intervention strategies

Keywords: schizophrenia, smoking, homosexuality, prisoners, prostitution, tobacco.

\section{Introducción}

La exclusión social ha sido reconocida por muchos años como un importante determinante de salud $(1,2)$. Son muchos los factores de riesgo, entre ellos los estilos de vida poco saludables, que llevan a mayor morbilidad y mortalidad en las poblaciones socialmente excluidas o discriminadas (3). El término "estigma" se ha definido como "atributo asociado con un descrédito profundo" (4), y son razones culturalmente poderosas de exclusión social. Existen numerosas formas de estigmas: los relacionados con la raza, edad o religión, varían según las circunstancias locales (5); otros, sin embargo, tienden a ser universales. Es el caso de los trastornos mentales (6), la conducta antisocial (o la reclusión en instituciones penitenciarias) (7), la preferencia sexual homosexual (la pertenencia a los grupos LGBTI: lesbianas, gay, bisexuales, transexuales e intersexuales) $(8,9)$ o la prostitución (10).

La investigación sobre la asociación de fumar con trastornos mentales fue iniciada por Weissman (11), y ha sido objeto de bastante investigación (12, 13). Se ha propuesto que las alteraciones del perfil lipídico inducidas por la medicación, así como el tabaquismo podrían estar relacionados con la menor expectativa de vida que se ha descrito en las principales enfermedades psiquiátricas $(14,15)$. La literatura sobre la asociación entre tabaquismo y conducta antisocial se ha centrado no sólo en las altas tasas de consumo de tabaco en la población carcelaria (16), sino en el consumo de cigarrillo como un predictor temprano de la personalidad delincuencial en niños y adolescentes (17). Varios estudios, en diferentes lugares del mundo, sugieren también un mayor consumo de cigarrillo en población tanto de lesbianas como de hombres gay $(18,19)$, y el hecho de fumar cigarrillos hace parte del estereotipo común de las trabajadoras sexuales (20).

El objetivo de este trabajo, que surgió a partir de un taller de búsquedas en PubMed y por tanto se limitó a esa base de datos, fue revisar de forma sistemática la literatura indexada en Medline (empleando el portal www.pubmed.com) sobre el consumo de tabaco en estas cuatro poblaciones específicas, para cuantificar la proporción de incremento del consumo y hacer comparaciones entre ellas.

\section{Materiales y métodos}

\section{Estrategia de búsqueda}

Todas las búsquedas se hicieron exclusivamente en la base de datos de Medline (www.pubmed.com) y se realizaron en noviembre de 2013. Para los artículos referentes al consumo de cigarrillo, en las cuatro búsquedas se emplearon los siguientes términos:

"tobacco"[mesh] OR "smoking"[mesh] OR "smoking cessation"[mesh] OR "tobacco Use disorder"[mesh].

Esta búsqueda, a su vez, se unió empleando el conector booleano "AND" con cada una de las siguientes cuatro frases (que además se unían con el conector "OR"):

- "schizophrenia"[mesh]

- "gay OR lesbian OR bisexual OR transgender OR queer OR LGBT OR homosex* OR transvest*"

- "penitent* OR prisoner OR hostage OR convict*

- “((sexual OR sex) AND (commercial OR workers)) OR prostitut* OR brothel" 
Se realizó un primer filtro, en el cual dos personas del equipo (cada uno por separado) revisaron los títulos y resúmenes (abstracts) de cada uno de los artículos, para eliminar aquellos claramente irrelevantes (aquellos que no indicaran prevalencias de consumo de tabaco en los grupos de interés). Cuando no había acuerdo entre los dos, se llegó a decisión mediante consenso.

Luego se hizo una revisión de los textos completos de los artículos restantes, de donde se extrajeron los datos sobre prevalencia de consumo de cigarri1lo, expresados en porcentaje. Cada vez que un grupo control fue mencionado en el estudio, se utilizó esa cifra para estimar las razones de disparidad; si la publicación no incluía un grupo control, se utilizaron para las comparaciones las cifras de prevalencia del Informe de la Organización Mundial de la Salud (OMS) sobre la Epidemia Mundial de Tabaquismo (disponible en http://www.who.int/tobacco/surveillance/policy/country_profile/en/). La heterogeneidad de los estudios incluidos se evaluó mediante la prueba de chi $^{2}$ y se hizo un análisis de las estrategias de muestreo para evaluar sesgos.

\section{Resultados}

La figura 1 muestra el proceso general para la recolección de datos en los 206 artículos finales. El total de artículos para cada una de las cuatro poblaciones consideradas fue: pacientes con esquizofrenia 112; personas con conducta homosexual 57, prisioneros 31 y trabajadores sexuales 6. De los 91 estudios no conseguidos, 34 eran anteriores a 1998 (fecha de introducción de los archivos en pdf).

\section{Pacientes con esquizofrenia}

Se identificaron en total 101 estudios hechos sobre un total de 46.912 pacientes (de los cuales 26.162, $55,8 \%$, eran fumadores). Los estudios provienen de 29 países (Alemania, Australia, Canadá, Chile, China, Colombia, Egipto, Escocia, España, Estados Unidos, Finlandia, Francia, Grecia, Hungría, India, Irlanda, Israel, Japón, Noruega, Reino Unido, Singapur, Suecia, Suiza, Taiwán, Túnez y Turquía). La prevalencia más baja de consumo es la reportada por Tang en China entre mujeres hospitalizadas con esquizofrenia, con una sola fumadora entre 78 mujeres (en contraste con los 50 hombres fumadores entre 116) (21). Hay también prevalencias bajas en India

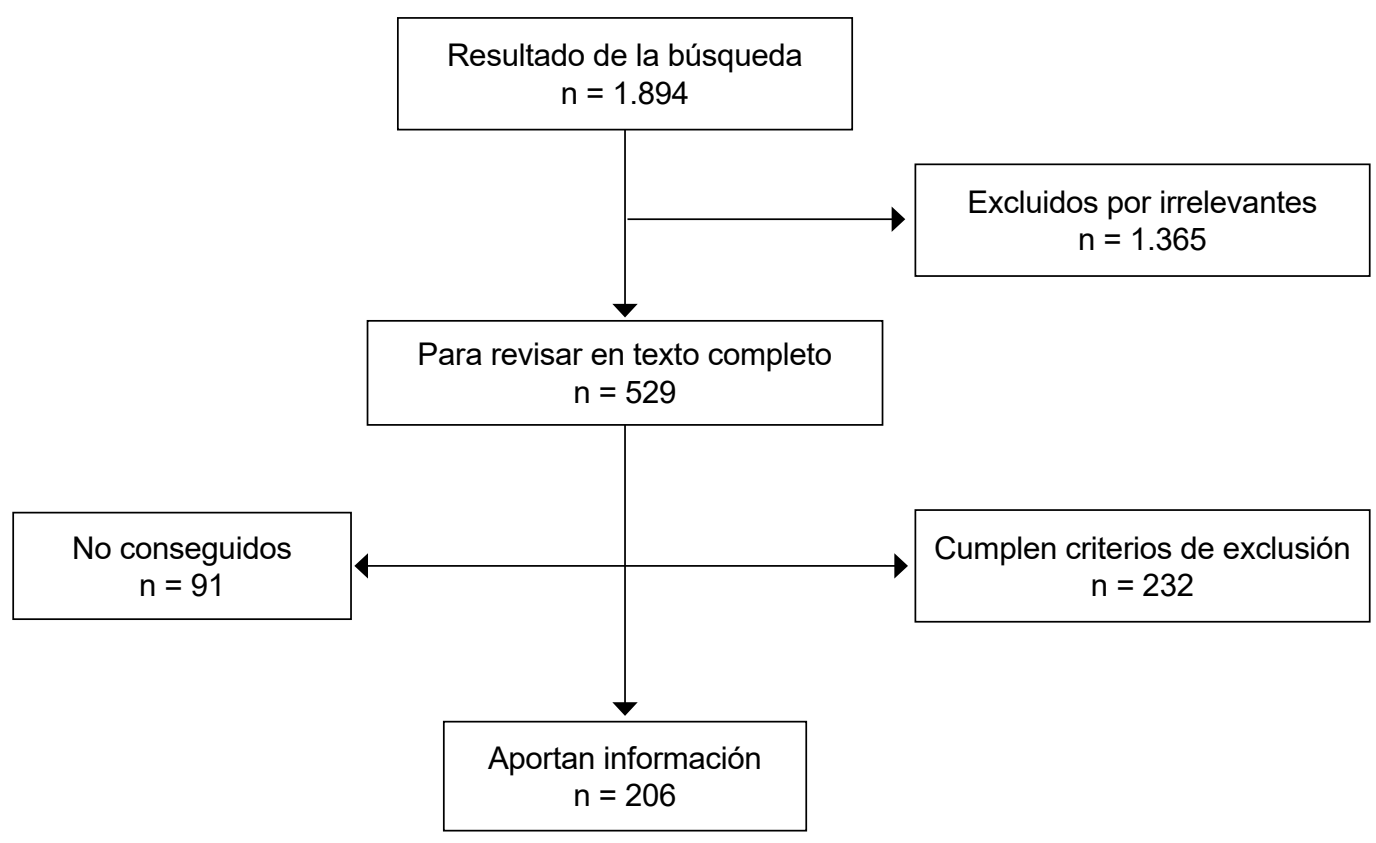

Figura 1. Proceso de selección de los artículos que aportan datos al estudio. 
(22), en Japón (23) y en el estudio de Campo-Arias (24) en mujeres en Bucaramanga (7 de 33 mujeres). Las prevalencias más elevadas se describen en Estados Unidos $(25,26)$ y en Irlanda (27), con algunas cifras de prevalencia cercanas al 90\%. Los resultados no son muy diferentes, con más fumadores en el sexo masculino, en los estudios hechos tanto sobre pacientes hospitalizados como pacientes ambulatorios. Con la excepción del estudio de Masterson (27) en Irlanda, que encontró prevalencia similares en hombres y mujeres ( 84 y $82 \%$, respectivamente), todos los otros estudios muestran un mayor consumo en hombres que en mujeres. Es el caso de Canadá (28), con $73,7 \%$ en hombres y $65,5 \%$ en mujeres; Finlandia (29) con $69,7 \%$ y $36,8 \%$, respectivamente; y sobre todo China, con 52,0\% de hombres fumadores, comparado con 4,5\% en mujeres (30). En resumen, aunque la heterogeneidad de las muestras no permite un metaanálisis de los datos, el promedio ponderado de la prevalencia de consumo de los sujetos de estos estudios es de 55,8\%, comparados con un $26,3 \%$ en los grupos control, para un OR de 3,34 (IC 95\% 2,95 - 3,78; chi $^{2}=1552$; grados de libertad $117 ; \mathrm{p}<0,00001 ; \mathrm{I}^{2}=92 \%$ ).

\section{Población homosexual}

Aunque la búsqueda estuvo orientada a buscar también transexuales e intersexuales, de estos dos grupos no se encontraron estudios, por lo que se reportan los resultados de hombres y mujeres homosexuales y bisexuales. En total, se extrajo información de 68 estudios realizados en 8 países (Australia, Canadá, China, Estados Unidos, Francia, Irlanda, México, Reino Unido y Suiza), aunque 57 de ellos (casi 85\%) fueron hechos en Estados Unidos. En total, el estudio incluyó 68.933 sujetos homosexuales o bisexuales (31.415 mujeres, 21.223 hombres, en los otros estudios aparecen agrupados hombres y mujeres). El OR para mujeres lesbianas y bisexuales fue de 2,21 (IC 95\% 1,84 $-2,65 ; \mathrm{chi}^{2}=901$; grados de libertad 52; $<<0,00001$; $\mathrm{I}^{2}=94 \%$ ) y para hombres 1,82 (IC 95\% 1,55 - 2,12; chi $^{2}=398$; grados de libertad $41 ; \mathrm{p}<0,00001 ; \mathrm{I}^{2}=$ 90\%). Para hombres y mujeres agrupados fue de 2,03 (IC 95\% 1,84-2,26; $\mathrm{chi}^{2}=1435$; grados de libertad $\left.109 ; \mathrm{p}<0,00001 ; \mathrm{I}^{2}=92 \%\right)$. Las prevalencias más altas $(79,7 \%)$ fueron encontradas entre hombres gay en
Suiza (31), así como entre lesbianas hispanas y afroamericanas pobres de Bronx (73,3\%) (32). También se encontró un alto consumo $(70,5 \%)$ entre hombres adolescentes y adultos jóvenes en una muestra poblacional de la ciudad de Nueva York (33), así como en una muestra similar $(66,6 \%)$ en Minnesota (34). El estudio más grande en mujeres lesbianas y bisexuales es mexicano (35), con una muestra de 7.245 mujeres, de las cuales 2.239 eran fumadoras, para un 30,9\% que, al comparar con la población femenina control de $11,4 \%$, arroja un OR de 3,48 (IC 95\% 3,08 - 3,80). El mismo autor estudia una muestra de 5.521 hombres homosexuales y bisexuales de México, y encuentra que $2.225(40,3 \%)$ son fumadores, en contraste con $30,0 \%$ en el grupo control.

\section{Reclusos}

Se identificaron 31 estudios realizados en 11 países, 13 de ellos en Estados Unidos y el resto en Australia, Canadá, China, Grecia, Irlanda, Lituania, México, Pakistán, Reino Unido y Suiza, con una muestra total de 22.414 presidiarios. En todas las cárceles estudiadas, los fumadores son mayoría, excepto en una cárcel de mujeres de Dakota del Sur (36) que era "libre de humo" (y sin embargo 24\% de las reclusas fumaba). De otro lado, las prevalencias de consumo de 90 \% y más, son relativamente comunes tanto en cárceles de hombres (37-39) como de mujeres (40). Los estudios que analizaron exclusivamente población masculina, encontraron una prevalencia total de 76,4 $\%$ (6.761 de 8.852), mientras que los que estudiaron exclusivamente población femenina encontraron una de 58,3\% (6.503 de 11.155). Al contrastar esos datos con la población de los respectivos grupos control, se encuentra que el odds ratio para hombres es de 6,60 (IC 95\% 5,15 - 8,46; chi $^{2}=177$; grados de libertad $17 ; \mathrm{p}<0,00001 ; \mathrm{I}^{2}=90 \%$ ) y el de mujeres de 9,72 (IC 95\% 5,95 - 15,90; chi $^{2}=361$; grados de libertad 11; $\left.\mathrm{p}<0,00001 ; \mathrm{I}^{2}=97 \%\right)$.

\section{Trabajadores sexuales}

Dos de los seis estudios analizados eran de Venezuela $(41,42)$ y se basaron en la misma muestra de 438 trabajadoras sexuales, 73,4\% de las cuales eran fumadoras (el promedio de consumo de la mujer 
en Venezuela es 27,0\%). Otro estudio provenía de China (43) y analizaba 532 mujeres que emigraron de las zonas rurales a las grandes ciudades chinas. El porcentaje de fumadores fue menor $(3,2 \%$ de 2.229) en las mujeres que trabajaban en hoteles o restaurantes, en comparación con las trabajadoras sexuales (41,9\% de 532). Un estudio de Corea del Sur (44) con 113 mujeres recuperadas de la prostitución, encontró que $90,2 \%$ de ellas eran fumadoras (prevalencia femenina en Corea 5,7 \% ). Berg (45), en Shanghái, estudió a 200 hombres trabajadores sexuales y 140 de ellos (70\%) eran fumadores activos. Por último, un estudio francés (46) con una muestra de 252 trabajadores sexuales masculinos encontró una prevalencia de tabaquismo de 75,0\% (frente al 36,6\% en los hombres franceses). El odds ratio para los trabajadores sexuales femeninos sería entonces 7,5 en Venezuela, 21,8 en China y 152,2 en Corea del Sur, para un promedio de 26,3 (IC 95\% 6,7 - 103,2; chi $^{2}=37$; grados de libertad $2 ; \mathrm{p}<0,00001 ; \mathrm{I}^{2}=95 \%$ en mujeres trabajadoras sexuales, y 5,2 (IC 95\% 3,9 - 6,9; chi $^{2}=0$; grados de libertad $1 ; \mathrm{p}=0,99 ; \mathrm{I}^{2}=0 \%$ ) en trabajadores sexuales masculinos.

\section{Discusión}

En este trabajo se escogieron cuatro grupos poblacionales estigmatizados, por causas diferentes, relevantes para la realidad colombiana actual; se dejaron por fuera muchas otras posibles causas de estigma (raciales, religiosos y muchos más). Los grupos seleccionados tienen en común que el estigma existe en todas las culturas. En todos ellos se encontraron, tasas elevadas de consumo de tabaco, algo previamente descrito en la literatura, pero aquí, de alguna forma, cuantificado. El metaanálisis más grande reportado en la literatura en pacientes con esquizofrenia, por ejemplo, incluía 42 estudios (13), en contraste con los 101 que aquí se han revisado aquí. Vale aclarar que en ese caso la búsqueda también se limitó exclusivamente a la base de datos de PubMed, pero incluyó solo estudios poblacionales y no los de pacientes institucionalizados. Los estudios en salud mental sugieren que los pacientes con esquizofrenia fuman más que la población general desde antes de la aparición de la psicosis, y además tienen unas tasas de suspensión más bajas que la población general (47). La literatura es consistente en hombres o mujeres, en pacientes ambulatorios o institucionales. Se ha sugerido incluso que el cigarrillo en estos pacientes con esquizofrenia es un predictor de suicidio (48). Los estudios en población LGBTI son más escasos, se concentran en subgrupos de pacientes (por ejemplo aquellos con VIH/SIDA), y en muchos casos la muestra es escogida en bares, lo que podría muy seguramente incidir en las altas tasas de consumo de tabaco. En presidiarios, el sesgo podría actuar en sentido contrario, ya que varios estudios fueron hechos en cárceles "libres de humo". La definición de "fumador" varía también de un estudio a otro, lo que se convierte en una limitación para la comparabilidad.

Una limitación adicional de este análisis es que, como en el metaanálisis de De León et al. (13), se limitó a una única base de datos. Haber incorporado otras, como Embase o SciELO, habría enriquecido el análisis y quizás habría aportado más información local o regional.

El consumo de tabaco en los cuatro grupos estudiados hace parte de otros rasgos de estilo de vida poco saludables, causantes de una mayor morbilidad y mortalidad. Las intervenciones de prevención de consumo o de cesación de su uso, en cada grupo poblacional deben estar diseñadas y dirigidas al grupo específico, si han de ser exitosas. Aunque no encontramos trabajos dirigidos a trabajadoras sexuales, sí hay experiencia en intervenciones para dejar de fumar en pacientes con esquizofrenia (4951), en presidiarios (52) y en la población LGBTI (53-55). Con la excepción de los pacientes con esquizofrenia, que han sido estudiados en Colombia $(24,56)$, los estilos de vida en los otros grupos poblacionales no han sido investigados en nuestro país; de hecho estos otros grupos con estigma han recibido poca atención en la investigación nacional. Los autores esperamos que este trabajo ayude a despertar ese interés.

\section{Conflictos de interés}

Los autores declaran no tener conflictos de interés. 


\section{Bibliografía}

1. Marmot M. Social determinants of health inequalities. Lancet. 2005;365:1099-104.

2. Wilkinson RG, Marmot MG. Social determinants of health: the solid facts. Geneva: World Health Organization; 2003.

3. Siegrist J. Place, social exchange and health: proposed sociological framework. Soc Sci Med. 2000;51:1283-93.

4. Goffman E. Stigma: Notes on the management of spoiled identity. New York: Simon \& Schuster; 1963.

5. Link BG, Phelan JC. Conceptualizing Stigma. Ann Rev Sociol. 2001;27:363-85.

6. Hayward P, Bright JA. Stigma and mental illness: A review and critique. J Mental Health. 1997;6:345-54.

7. Rasmusen EB. Stigma and self-fulfilling expectations of criminality. J Law Econ. 1996;39:519-44.

8. Herek GM. Stigma and sexual orientation: Understanding prejudice against lesbians, gay men, and bisexuals. Herek GM, editor. Thousand Oaks, CA, US: Sage Publications, Inc; 1998. X. p. 278.

9. Herek GM. Beyond "Homophobia": Thinking about sexual prejudice and stigma in the twenty-first century. Sex Res Social Pol. 2004;1:6-24.

10. Ward H, Aral SO. Globalisation, the sex industry, and health. Sex Transm Infect. 2006;82:345-7.

11. Weissman MM, Klerman GL. Epidemiology of mental disorders: emerging trends in the United States. Arch Gen Psychiatry. 1978;35:705-12.

12. Goff DC, Henderson DC, Amico E. Cigarette smoking in schizophrenia: relationship to psychopathology and medication side effects. Am J Psychiatry. 1992;149:1189-94.

13. de Leon J, Diaz FJ. A meta-analysis of worldwide studies demonstrates an association between schizophrenia and tobacco smoking behaviors. Schizophr Res. 2005;76(-3):135-57.

14. Colton CW, Manderscheid RW. Congruencies in increased mortality rates, years of potential life lost, and causes of death among public mental health clients in eight states. Prev Chronic Dis. 2006;3:A42.

15. Dembling BP, Chen DT, Vachon L. Life expectancy and causes of death in a population treated for serious mental illness. Psychiatr Serv. 1999;50:1036-42.

16. Binswanger IA, Krueger PM, Steiner JF. Prevalence of chronic medical conditions among jail and prison inmates in the USA compared with the general population. J Epidemiol Community Health. 2009;63:912-9.

17. Farrington DP. Early predictors of adolescent aggression and adult violence. Violence Vict. 1989;4:79-100.

18. Stall RD, Greenwood GL, Acree M, Paul J, Coates TJ. Cigarette smoking among gay and bisexual men. Am J Public Health. 1999;89:1875-8.
19. Ryan H, Wortley PM, Easton A, Pederson L, Greenwood G. Smoking among lesbians, gays, and bisexuals: a review of the literature. Am J Prev Med. 2001;21:142-9.

20. Sterk CE, Elifson KW. Drug-related violence and street prostitution. NIDA Res Monogr 1990;103:208-21.

21. Tang YL, Mao P, Li FM, Li W, Chen Q, Jiang F, et al. Gender, age, smoking behaviour and plasma clozapine concentrations in 193 Chinese inpatients with schizophrenia. Br J Clin Pharmacol. 2007;64:49-56.

22. Srinivasan TN, Thara R. Smoking in schizophrenia -- all is not biological. Schizophr Res. 2002;56:67-74.

23. Mori T, Sasaki T, Iwanami A, Araki T, Mizuno K, Kato T, et al. Smoking habits in Japanese patients with schizophrenia. Psychiatry Res. 2003;120:207-9.

24. Campo-Arias A, Díaz-Martínez LA, Rueda-Jaimes GE, RuedaSánchez M, Farelo-Palacín D, Diaz FJ, et al. Smoking is associated with schizophrenia, but not with mood disorders, within a population with low smoking rates: a matched case-control study in Bucaramanga, Colombia. Schizophr Res. 2006;83:269-76.

25. Hughes JR, Hatsukami DK, Mitchell JE, Dahlgren LA. Prevalence of smoking among psychiatric outpatients. Am J Psychiatry. 1986;143:993-7.

26. Diwan A, Castine M, Pomerleau CS, Meador-Woodruff JH, Dalack GW. Differential prevalence of cigarette smoking in patients with schizophrenic vs mood disorders. Schizophr Res. 1998;33:113-8.

27. Masterson E, O'Shea B. Smoking and malignancy in schizophrenia. Br J Psychiatry. 1984;145:429-32.

28. Cohn T, Prud'homme D, Streiner D, Kameh H, Remington G. Characterizing coronary heart disease risk in chronic schizophrenia: high prevalence of the metabolic syndrome. Can J Psychiatry. 2004;49:753-60.

29. Salokangas RK, Saarijärvi S, Taiminen T, Lehto H, Niemi H, Ahola V, et al. Effect of smoking on neuroleptics in schizophrenia. Schizophr Res. 1997;23:55-60.

30. Tang YL, Gillespie CF, Epstein MP, Mao PX, Jiang F, Chen Q, et al. Gender differences in 542 Chinese inpatients with schizophrenia. Schizophr Res. 2007;97(-3):88-96.

31. Schwappach DL. Queer quit: gay smokers' perspectives on a culturally specific smoking cessation service. Health Expect. 2009; 12:383-95.

32. Sanchez JP, Meacher P, Beil R. Cigarette smoking and lesbian and bisexual women in the Bronx. J Community Health. 2005;30:23-37.

33. Storholm ED, Halkitis PN, Siconolfi DE, Moeller RW. Cigarette smoking as part of a syndemic among young men who have sex with men ages 13-29 in New York City. J Urban Health. 2011;88:663-76.

34. Remafedi G, Carol H. Preventing tobacco use among lesbian, gay, bisexual, and transgender youths. Nicotine Tob Res. 2005; 7:249-56. 
35. Ortiz-Hernández L, Tello BL, Valdés J. The association of sexual orientation with self-rated health, and cigarette and alcohol use in Mexican adolescents and youths. Soc Sci Med. 2009;69:85-93.

36. Khavjou OA, Clarke J, Hofeldt RM, Lihs P, Loo RK, Prabhu $\mathrm{M}$, et al. A captive audience: bringing the WISEWOMAN program to South Dakota prisoners. Womens Health Issues. 2007; 17:193-201.

37. Skondras M, Markianos M, Botsis A, Bistolaki E, Christodoulou G. Platelet monoamine oxidase activity and psychometric correlates in male violent offenders imprisoned for homicide or other violent acts. Eur Arch Psychiatry Clin Neurosci. 2004;254:380-6.

38. Lekka NP, Lee KH, Argyriou AA, Beratis S, Parks RW. Association of cigarette smoking and depressive symptoms in a forensic population. Depress Anxiety. 2007;24:325-30.

39. Voglewede JP, Noel NE. Predictors of current need to smoke in inmates of a smoke-free jail. Addict Behav. 2004;29:343-8.

40. Durrah TL. Correlates of daily smoking among female arrestees in New York City and Los Angeles, 1997. Am J Public Health. 2005;95:1788-92.

41. Núñez JT, Delgado M, Pino G, Girón H, Bolet B. Smoking as a risk factor for preinvasive and invasive cervical lesions in female sex workers in Venezuela. Int J Gynaecol Obstet. 2002;79:57-60.

42. Núñez JT, Delgado M, Girón H, Pino G. Prostitution and other cofactors in preinvasive and invasive lesions of the cervix. Aust N Z J Obstet Gynaecol. 2004;44:239-43.

43. Wan X, Shin SS, Wang Q, Raymond HF, Liu H, Ding D, et al. Smoking among young rural to urban migrant women in China: a cross-sectional survey. PLoS One. 2011;6:e23028.

44. Jung YE, Song JM, Chong J, Seo HJ, Chae JH. Symptoms of posttraumatic stress disorder and mental health in women who escaped prostitution and helping activists in shelters. Yonsei Med J. 2008;49:372-82.

45. Berg CJ, Nehl EJ, Wong FY, He N, Huang ZJ, Ahluwalia JS, et al. Prevalence and correlates of tobacco use among a sample of MSM in Shanghai, China. Nicotine Tob Res. 2011;13:22-8.
46. Laurindo da Silva L. Les modalités de consommation de drogues chez les prostitues masculins. Sante Publique. 2006;18:207-21.

47. de Leon J. Smoking and vulnerability for schizophrenia. Schizophr Bull. 1996;22:405-9.

48. Iancu I, Sapir AP, Shaked G, Poreh A, Dannon PN, Chelben $\mathrm{J}$, et al. Increased suicidal risk among smoking schizophrenia patients. Clin Neuropharmacol. 2006;29:230-7.

49. Addington J, el-Guebaly N, Campbell W, Hodgins DC, Addington D. Smoking cessation treatment for patients with schizophrenia. Am J Psychiatry. 1998;155:974-6.

50. Tsoi DT, Porwal M, Webster AC. Interventions for smoking cessation and reduction in individuals with schizophrenia. Cochrane Database Syst Rev. 2013;2:CD007253.

51. Robson D, Cole F, Jalasi S, Boojharut B, Smith S, Thompson $\mathrm{S}$, et al. Smoking cessation and serious mental illness: a service evaluation of a drop-in stop smoking clinic on an acute in-patient unit. J Clin Nurs. 2013;22:405-13.

52. Berg CJ, Ahluwalia JS, Cropsey K. Predictors of adherence to behavioral counseling and medication among female prisoners enrolled in a smoking cessation trial. J Correct Health Care. 2013;19:236-47.

53. Dickson-Spillmann M, Sullivan R, Zahno B, Schaub MP. Queer quit: a pilot study of a smoking cessation programme tailored to gay men. BMC Public Health. 2014;14:126.

54. Grady ES, Humfleet GL, Delucchi KL, Reus VI, Muñoz RF, Hall SM. Smoking cessation outcomes among sexual and gender minority and nonminority smokers in extended smoking treatments. Nicotine Tob Res 2014 Apr 11. [Epub ahead of print]

55. Matthews AK, Li CC, Kuhns LM, Tasker TB, Cesario JA. Results from a community-based smoking cessation treatment program for LGBT smokers. J Environ Public Health. 2013;2013:984508. doi: 10.1155/2013/984508.

56. Diaz FJ, Velásquez DM, Susce MT, de Leon J. The association between schizophrenia and smoking: unexplained by either the illness or the prodromal period. Schizophr Res. 2008; 104:214-9. 